\title{
Avaliação antropométrica de crianças de escolas públicas e particulares em um município do interior paulista
}

\author{
Anthropometric evaluation of children in public and private schools in a municipality from \\ São Paulo interior
}
Evaluación antropométrica de niños de escuelas públicas y particulars en un municipio del interior paulista

Humberto Tavares de Carvalho Júnior ${ }^{1}$, Roberta Alves de Macedo ${ }^{1}$, Miguel Murata ${ }^{1}$, Willian Cesar Pena Soares da Silva ${ }^{1}$, Maíra Ferro de Sousa Touso ${ }^{1 *}$.

\section{RESUMO}

Objetivo: Comparar dados antropométricos, hábitos alimentares e frequência de esportes em crianças de uma rede pública e particular de ensino. Métodos: Participaram do estudo 80 crianças, com faixa etária de 7 a 10 anos, na qual 35 estavam na escola pública e 45 em uma escola particular. Foram coletados os dados antropométricos peso, altura e calculado o índice de massa corporal. Posteriormente, foi aplicado um questionário em cada participante sobre a qualidade de seus hábitos alimentares e prática de esportes. Foi utilizado o teste t de Student para comparação de dois grupos independentes; e, nos dados em que estiveram envolvidos mais que dois grupos, foi realizada a Análise de Variância de Fisher (ANOVA) com pós-teste de Tukey, com nível de significância de 5,0\% $(\alpha=0,05)$. Resultados: A proporção de crianças que praticam esportes $\left(p=0,0060^{\star}\right)$ e a frequência dessa prática $\left(p=0,003^{*}\right)$ são maiores nos escolares da escola particular; as crianças das escolas públicas apresentaram maior proporção de refeições frente à televisão $\left(p=0,0026^{*}\right)$. Conclusão: Estes achados demonstram características de hábitos de saúde, relevantes para o crescimento e desenvolvimento da criança, que apresentam diferenças entre as redes de ensino pública e particular, ressaltando que a rede pública deve priorizar mudanças para minimizar possíveis hábitos que comprometerão a criança pela sua vida.

Palavras chaves: Criança, Desenvolvimento infantil, Antropometria.

\begin{abstract}
Objective: To compare anthropometric data, eating habits and sports frequency in children in a public and private school network. Methods: 80 children participated in this study, with ages ranging from 7 to 10 years, which 35 were from public school and 45 in a private school. Were collected anthropometric data, weight, height and calculated the body mass index. Subsequently, a questionnaire was applied to each participant about quality of their eating habits and sports practice. Student's t-test was used to compare two independent groups; and in the data which more than two groups were involved, Fisher's Variance Analysis (ANOVA) with Tukey post-test was performed, with a significance level of $5.0 \%(\alpha=0.05)$. Results: The proportion of children who practice sports $\left(p=0.0060^{*}\right)$ and the frequency of this practice $\left(p=0.003^{*}\right)$ are higher among schoolchildren in private school; children in public schools had a higher proportion of meals in front of television $\left(p=0.0026^{*}\right)$. Conclusion: These findings demonstrate characteristics of health habits, relevant to child's growth and development, that present differences between public and private education networks, emphasizing public network must prioritize changes to minimize possible habits that will compromise the child for his or her life.
\end{abstract}

Keywords: Child, Child development, Anthropometry.

${ }^{1}$ Faculdade de Medicina, Universidade de Franca (UNIFRAN). *E-mail: maira.touso@unifran.edu.br 


\section{RESUMEN}

Objetivo: Comparar datos antropométricos, hábitos alimenticios y frecuencia de deportes en niños de una red pública y particular de enseñanza. Métodos: Participaron 80 niños, con un grupo de edad de 7 a 10 años, 35 estaban en escuela pública y 45 en escuela particular. Se han recogido los datos antropométricos peso, altura y calculado el índice de masa corporal. Posteriormente, se aplicó un cuestionario en cada participante sobre la calidad de sus hábitos alimentarios y la práctica de deportes. Se utilizó la prueba t de Student para comparación de dos grupos independientes; y los datos en que estuvieron involucrados más de dos grupos, se realizó Análisis de Varianza de Fisher (ANOVA) con post-test Tukey, con nivel de significancia del 5,0\% $(\alpha=0,05)$. Resultados: La proporción de niños que practican deportes $\left(p=0,0060^{\star}\right)$ y la frecuencia desta práctica $\left(p=0,003^{*}\right)$ son mayores en los escolares de la escuela particular; los niños de la escuela pública presentaron mayor proporción de comidas frente a la televisión $\left(p=0,0026^{*}\right)$. Conclusión: Estos hallazgos demuestran características de hábitos de salud, relevantes para el crecimiento y desarrollo del niño, que presentan diferencias entre las redes de enseñanza pública y privada, resaltando que la red pública debe priorizar cambios para minimizar posibles hábitos que comprometerán al niño por su vida.

Palabras-clave: Niño, Desarrollo infantil,Antropometría.

\section{INTRODUÇÃO}

O estado nutricional de uma criança é consequência de vários fatores que se somam, de modo a possuir grande influência o meio em que esta vive. Nos países em desenvolvimento, como o Brasil, é necessária uma constante vigilância das condições nutricionais da população, dada a alta prevalência de distúrbios alimentares, tanto a desnutrição como, mais recentemente, a obesidade (RINALDI AEM, et al., 2008), que os dados mais recentes dos últimos 10 anos mostraram que houve aumento da prevalência da obesidade, de 11,8\% em 2006 para 18,9\% em 2016, atingindo quase um em cada cinco brasileiros (MINISTÉRIO DA SAÚDE, 2016).

A partir disso, é parte crucial do estudo de uma criança sua avaliação nutricional, pois os primeiros anos de vida são essenciais para o desenvolvimento. Com isso, pode-se observar se o crescimento está afastando do padrão esperado, devido a condições ambientais desfavoráveis ou a algum processo patológico (MINISTÉRIO DA SAÚDE, 2014).

O Brasil vive uma época de transição nutricional na qual as doenças e fatores que causam desnutrição e baixo desenvolvimento estão dando lugar a doenças e fatores que causam obesidade infantil e distúrbios metabólicos, devido às mudanças no estilo de vida, padrão dietético e inatividade física (MINISTÉRIO DA SAÚDE, 2014).

Com o aumento da urbanização, fator que leva à mudança nos padrões de morbimortalidade, hoje ocorre uma redução da mortalidade por doenças infectocontagiosas e aumento das doenças crônicas não transmissíveis (diabetes, dislipidemias, hipertensão arterial sistêmica e doenças cardiovasculares) (SANTOS DS, et al., 2019; CADAMURO SDP, et al., 2016).

Práticas de uma boa alimentação e esportes devem ser incentivadas e aprimoradas, principalmente na infância, já que muitas das concepções geradas nesta etapa serão levadas para a vida adulta (COLEONE JD, et al., 2017).

É sabido que após o processo de industrialização e urbanização, em conjunto à exposição excessiva de multimídias como televisores, computadores e jogos eletrônicos, houve diminuição da prática de esportes na faixa etária pediátrica.

O esporte em crianças melhora o perfil lipídico e metabólico, reduz a prevalência de obesidade na idade adulta, aumenta a probabilidade de tornar-se um adulto ativo, tendo como base sólida a diminuição do comportamento sedentário, condicionando uma melhor qualidade de vida (MITRE RMA e GOMES R, 2004). 
A orientação do esporte em crianças tem o propósito de criar o hábito e interesse, sem visar o desempenho desta. Assim, objetiva à inclusão no cotidiano, valorizando a educação física nas escolas através do esporte, que educa e socializa, promovendo sua saúde (MITRE RMA e GOMES R, 2004).

Devido a limitada acessibilidade e a escassez da prática orientada sobre esportes, apenas uma pequena parcela da população as realiza, devendo ser considerado diferentes fatores sóciodemográficos, ambientais e escolares como principais influenciadores sobre a prática (SILVA J, et al., 2016).

Além do incentivo à prática de esporte no período infantil, não se pode descartar a relevância do incentivo ao brincar. Essa fase do desenvolvimento humano é rica de pensamentos fantasiosos, manifestados pelas brincadeiras, pois cada criança partilha de uma cultura lúdica, a qual é formada a partir da introjeção de regras do meio social que a circunda (COSTA AR, BARROS TES e KUNZ E, 2018).

O brincar manifesta-se como uma possibilidade de expressão de sentimentos, preferências, receios e hábitos. Além disso, promove a interação da criança com seus semelhantes e contribui com o desenvolvimento físico, no caso de brincadeiras ativas (ANTUNES M e LUÍS H, 2018).

Compreender sobre a influência de fatores socioeconômicos e culturais na alimentação e prática de esportes das crianças mostra-se essencial, pois vivemos uma transição epidemiológica onde a obesidade e doenças cardiovasculares são relevantes, onde se faz necessário o estudo sobre os principais fatores que interferem e influenciam a prevalência destas desordens. Sendo assim, este estudo tem como objetivo comparar dados antropométricos, hábitos alimentares e frequência de esportes de crianças em uma rede pública de ensino e em uma rede particular, para identificar as possíveis implicações na área de saúde, afim de comparar as medidas obtidas entre si, estabelecendo uma relação entre a nutrição e prática de esportes das crianças nos diferentes níveis sociais.

\section{MÉTODOS}

Trata-se de um estudo transversal, quantitativo realizado em duas escolas de ensino fundamental, sendo uma escola pública e outra particular. O presente estudo foi encaminhado ao comitê de ética em pesquisa da Universidade de Franca e, somente após sua aprovação (CAAE: 99625218.5.0000.5495), os responsáveis pelas crianças, estudantes das escolas, que concordassem em tê-las participando do estudo assinaram o Termo de Consentimento Livre e Esclarecido.

Como critérios de inclusão deste estudo foram as crianças com faixa etária de 7 a 10 anos, matriculadas nas escolas participantes. Como critérios de não inclusão foram as crianças que se recusaram aos testes e aquelas cujos pais não autorizaram a participação. Desta forma, foram elegíveis para o estudo 35 crianças da escola pública e 45 da escola particular. Inicialmente, foram averiguados nos participantes os dados antropométricos (peso, altura e calculado o Índice de Massa Corporal - IMC, peso dividido pela altura ao quadrado).

Após a coleta dos dados antropométricos, foi aplicado um questionário nas crianças, na qual foi perguntado ao participante sobre seus hábitos alimentares e sua rotina de prática de esportes. O questionário sobre os hábitos alimentares, elaborado pelos autores, continha perguntas sobre quais alimentos elas consumiam no café da manhã, lanche, almoço e jantar, com intuito de definir a qualidade de suas refeições; além, também, de perguntas à respeito se elas faziam as refeições junto à família e se costumava fazer a refeição em frente à televisão.

O questionário sobre a prática de esportes da criança, também elaborado pelos autores, perguntava se os participantes praticavam algum esporte (se sim, qual e quantas vezes por semana) e sobre sua brincadeira preferida, com o intuito de determinar a influência destes fatores no estado nutricional.

Os dados antropométricos coletados foram analisados de acordo com as curvas de peso/idade, altura/idade, e cálculo do IMC, presentes na literatura e interpretados em seguida de acordo com os valores padrão (MINISTÉRIO DA SAÚDE, 2011). Os dados antropométricos e as respostas dos questionários foram tabulados em uma planilha eletrônica. 
As variáveis numéricas foram caracterizadas pelos parâmetros de média aritmética e desvio padrão. Em função do teste de D'Agostino e Pearson ter projetado populações de origem normalizadas, a condução da inferência estatística se fez na perspectiva paramétrica. Assim, para a comparação de dois grupos independentes foi utilizado o teste t de Student e, nos casos em que estiveram envolvidos na análise mais do que dois grupos os contrastes, se fizeram pela Análise de Variância de Fisher (ANOVA) com pós-teste de Tukey.

A medida de associação utilizada no caso de variáveis qualitativas foi a estatística de Qui-quadrado $\left(\mathrm{X}^{2}\right)$. Em todos os testes estatísticos o nível de significância pré-estabelecido foi de $5,0 \%(\alpha=0,05)$. Os cálculos foram realizados no software GraphPad Prism 5.0 e os gráficos descritivos, no software Excel do Office 2010 da Microsoft.

\section{RESULTADOS}

Participaram deste estudo 80 estudantes e seus dados sociodemográficos estão apresentados na Tabela 1.

Tabela 1- Dados sociodemográficos dos participantes.

\begin{tabular}{ccc}
\hline Variáveis & Escola pública & Escola particular \\
\hline Sexo feminino & $19(54 \%)$ & $25(55 \%)$ \\
Sexo masculino & $16(46 \%)$ & $20(45 \%)$ \\
Peso & $30.6 \pm 13$ & $33,3 \pm 3.5$ \\
Altura & $1.33 \pm 0.07$ & $1,4 \pm 0.06$ \\
Índice de Massa Corporal & $22 \pm 2.8$ & $18 \pm 2.8$ \\
\hline
\end{tabular}

Fonte: Dados da pesquisa, 2019.

Em relação a qualidade dos alimentos ingeridos pelas crianças durante as refeições, não foram encontradas diferenças significativas no consumo de carboidratos, proteínas, lipídios e fibras $(p>0,05)$ nos contrastes eutrófico $\mathrm{x}$ sobrepeso, eutrófico $\mathrm{x}$ obeso e sobrepeso $\mathrm{x}$ obeso no pós teste de Tukey, na ANOVA de Fischer, conforme Tabela 2.

Tabela 2 - Comparação entre qualidade de nutrientes ingeridos e perfil dos participantes segundo o IMC.

\begin{tabular}{|c|c|c|c|c|}
\hline Nutriente & Perfil & $\begin{array}{c}\text { Média } \pm \\
\text { D.Padrão }\end{array}$ & Signific & Cância \\
\hline \multirow{3}{*}{ Carboidratos } & eutrofia & $62,10 \pm 9,76$ & \multirow{3}{*}{$\begin{array}{l}\text { eutr x sobrep: } \\
\text { eutr x obes: } \\
\text { sobrep x obes: }\end{array}$} & \multirow{3}{*}{$\begin{array}{l}p<0,05(n s) \\
p<0,05(n s) \\
p<0,05(n s)\end{array}$} \\
\hline & sobrepeso & $57,18 \pm 8,41$ & & \\
\hline & obesidade & $55,04 \pm 13,24$ & & \\
\hline \multirow{3}{*}{ Proteínas } & eutrofia & $20,67 \pm 7,95$ & \multirow{3}{*}{$\begin{array}{l}\text { eutr x sobrep: } \\
\text { eutr x obes: } \\
\text { sobrep x obes: }\end{array}$} & \multirow{3}{*}{$\begin{array}{l}p<0,05 \text { (ns) } \\
p<0,05 \text { (ns) } \\
p<0,05 \text { (ns) }\end{array}$} \\
\hline & sobrepeso & $24,38 \pm 8,00$ & & \\
\hline & obesidade & $24,64 \pm 7,62$ & & \\
\hline \multirow{3}{*}{ Lipídios } & eutrofia & $4,43 \pm 3,59$ & \multirow{3}{*}{$\begin{array}{l}\text { eutr x sobrep: } \\
\text { eutr x obes: } \\
\text { sobrep x obes: }\end{array}$} & \multirow{3}{*}{$\begin{array}{l}p<0,05(n s) \\
p<0,05(n s) \\
p<0,05(n s)\end{array}$} \\
\hline & sobrepeso & $5,73 \pm 4,41$ & & \\
\hline & obesidade & $7,54 \pm 8,12$ & & \\
\hline \multirow{3}{*}{ Fibras } & eutrofia & $12,38 \pm 5,17$ & \multirow{3}{*}{$\begin{array}{l}\text { eutr x sobrep: } \\
\text { eutr x obes: } \\
\text { sobrep x obes: }\end{array}$} & \multirow{3}{*}{$\begin{array}{l}p<0,05(n s) \\
p<0,05(n s) \\
p<0,05(n s)\end{array}$} \\
\hline & sobrepeso & $11,71 \pm 6,58$ & & \\
\hline & obesidade & $13,83 \pm 9,96$ & & \\
\hline
\end{tabular}

Nota: eutr: eutrofia; ns: não significativo; obes: obesidade; sobrep: sobrepeso.

Fonte: Dados da pesquisa, 2019. 
Não há associação significativa entre escola e perfil antropométrico definido pelo IMC $\left(X^{2}=1,46 ; p=0,4820\right)$, conforme Gráfico 1.

Gráfico 1 - Relação da proporção dos estudantes segundo a classificação do IMC.

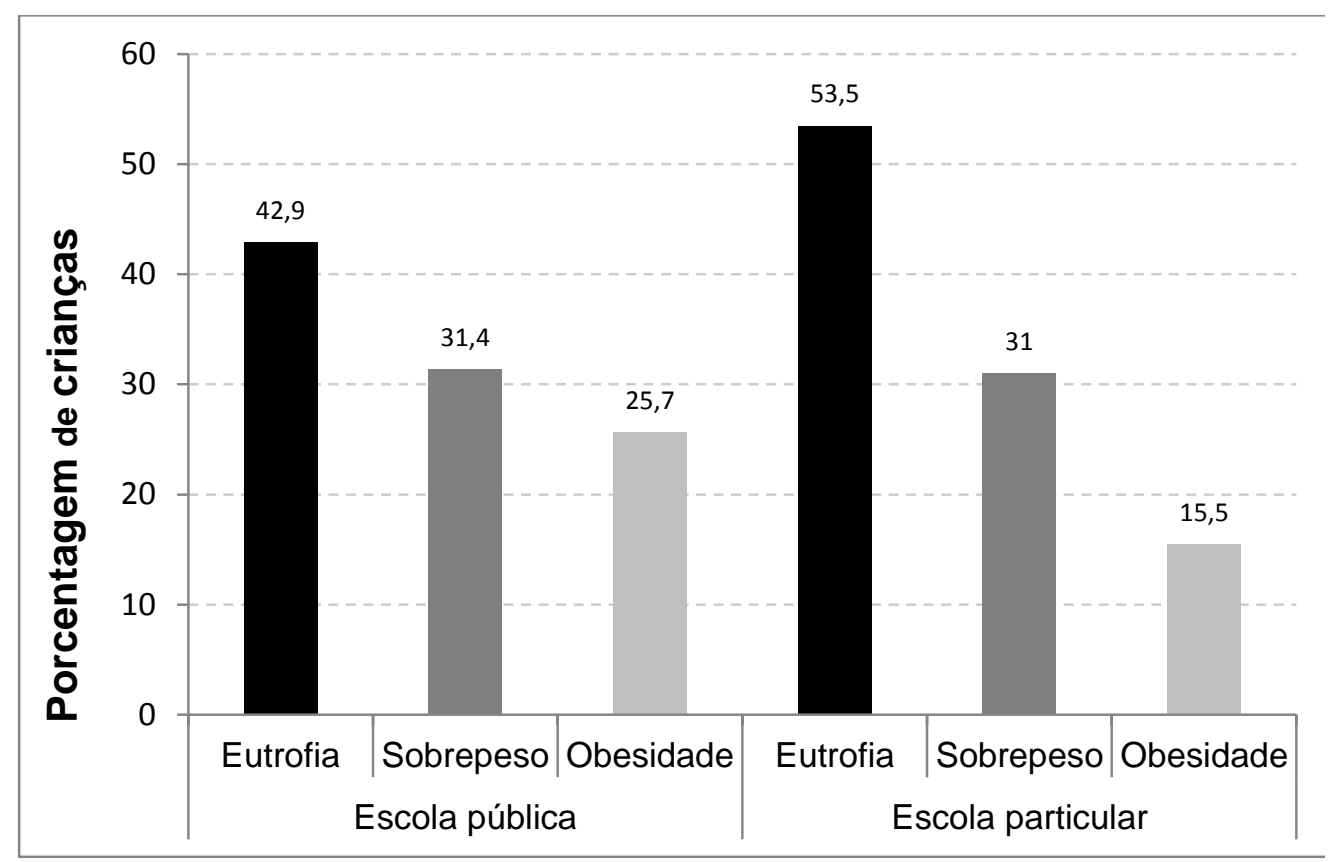

Fonte: Dados da pesquisa, 2019.

Quanto à proporção de crianças que fazem refeições em companhia dos pais, não há diferença estatisticamente significativa entre a escola pública $(85,7 \%)$ e a escola particular $(88,9 \%)(p=0,3351)$. A proporção de crianças que faz refeições frente à TV é significativamente maior na escola pública $(60,0 \%)$, comparada a essa proporção na escola particular $(28,9 \%)\left(p=0,0026^{\star}\right)$, conforme Tabela 2.

Gráfico 2 - Proporção de estudantes que fazem refeições com os pais e em frente à TV.

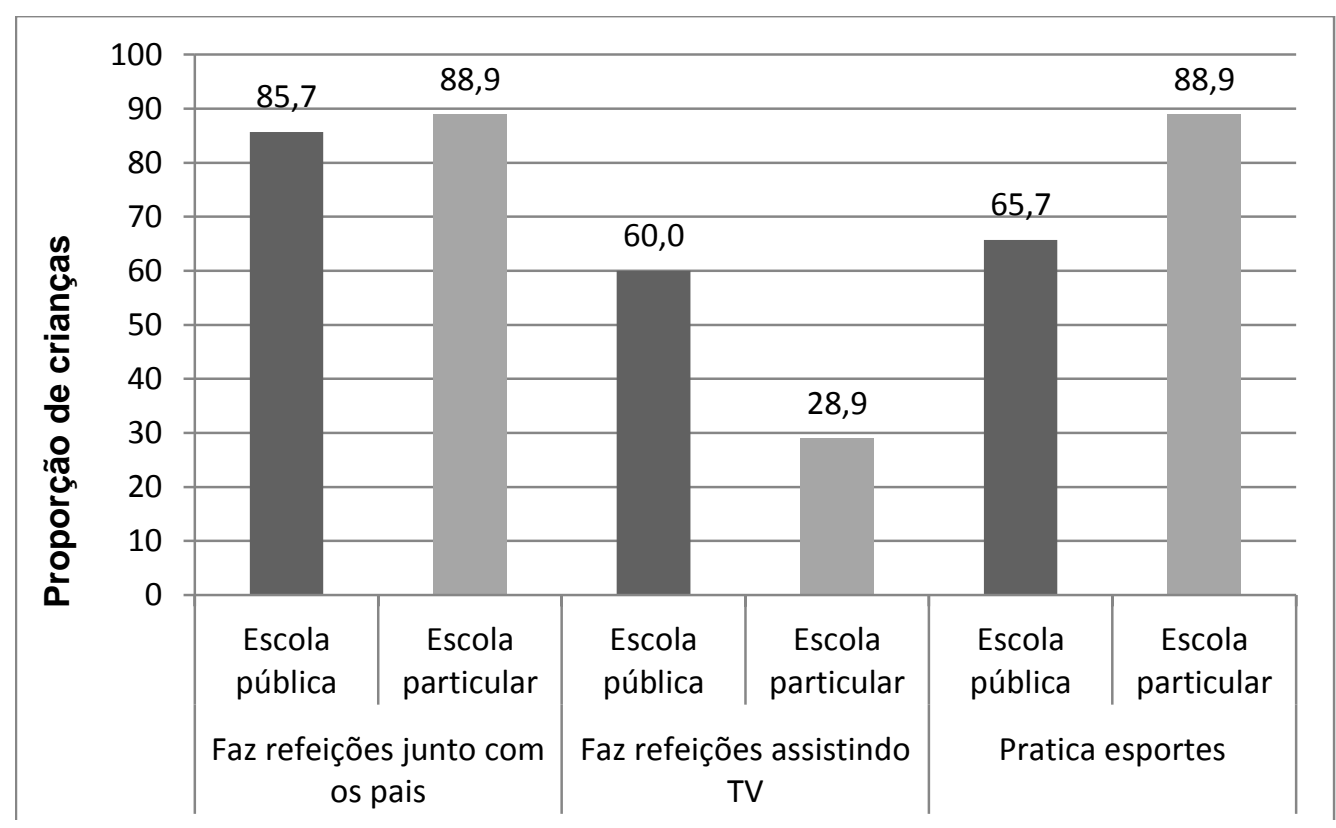

Fonte: Dados da pesquisa, 2019. 
A proporção de crianças que pratica esporte é significativamente menor na escola pública $(65,7 \%)$, comparada a essa proporção na escola particular $(88,9 \%)\left(p=0,0060^{*}\right)$, conforme Gráfico 2; a frequência semanal média dessa prática é também significativamente maior entre os alunos da escola particular por semana $2,80 \pm 1,85$ contra $1,42 \pm 1,32$ vezes por semana entre os alunos da escola pública $\left(p=0,003^{\star}\right)$, conforme Gráfico 3.

Gráfico 3 - Frequência média semanal de prática de atividade física.

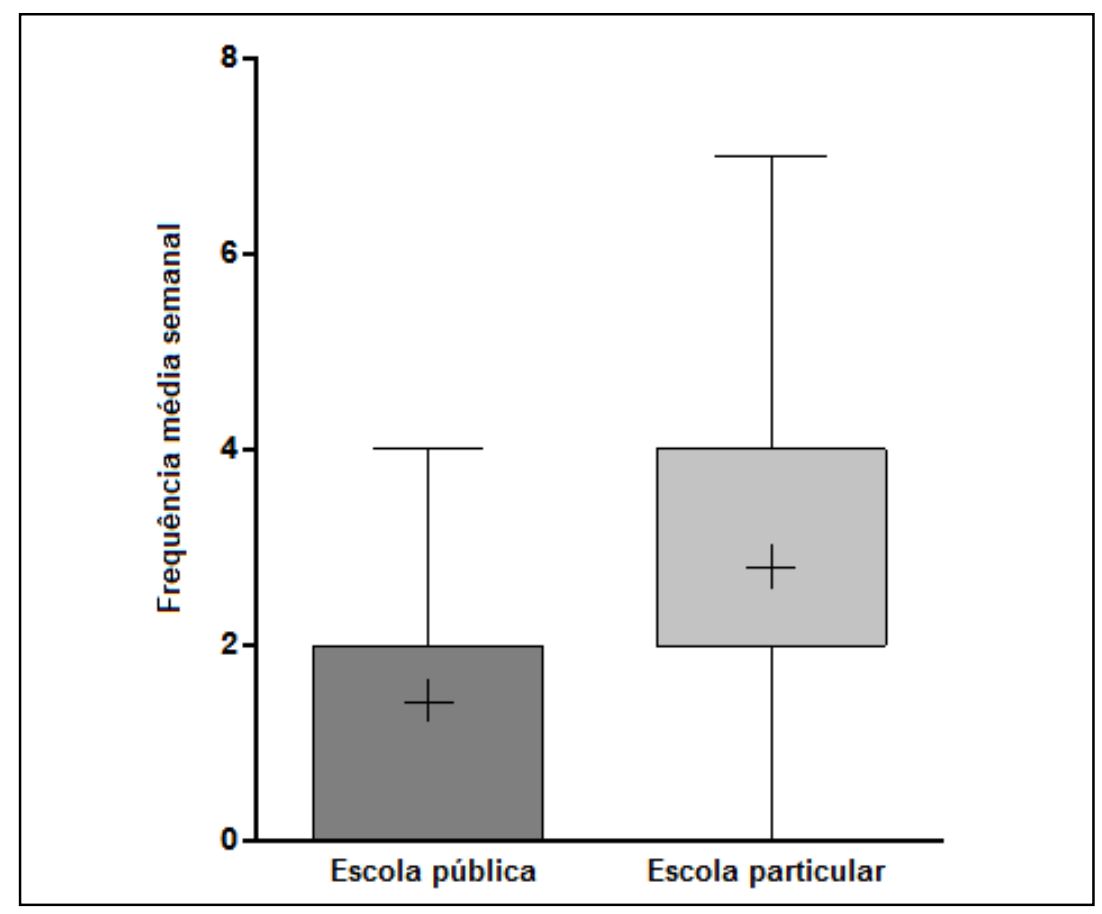

Fonte: Dados da pesquisa, 2019.

\section{DISCUSSÃO}

A detecção precoce de maus hábitos alimentares e não prática de esportes repercutem em inadequados índices antropométricos que permitem intervenção precoce, além de minimizar risco de desenvolvimento de doenças crônicas no futuro.

Neste estudo, foi possível verificar que 38 (47\%) dos participantes desta pesquisa são categorizados como eutróficos, sendo que $42.9 \%$ estão na escola pública e $53,5 \%$ são matriculados na escola particular. São dados positivos e condizentes com os achados à respeitos da qualidade dos alimentos que os estudantes ingerem, sendo possível verificar que não houve diferenças significativas no consumo de carboidratos, proteínas, lipídios e fibras nas comparações entre eutrófico $\mathrm{x}$ sobrepeso, eutrófico $\mathrm{x}$ obeso e sobrepeso $\mathrm{x}$ obeso.

Esses dados mostram que ações de educação alimentar e nutricional, previstas no Programa Nacional de Alimentação Escolar, estão sendo reforçadas a fim de promover melhora na situação alimentar e nutricional dos escolares (COLEONE JD, et al., 2017).

Estes achados demonstram que a classe social não é um fator que promove diferença nos hábitos alimentares das crianças, podendo estar associada a políticas públicas bem-sucedidas de distribuição de renda, erradicação da pobreza absoluta, ampliação do acesso a serviços básicos de saneamento, educação e saúde, um processo conhecido como transição epidemiológica (MINIESTÉRIO DA SAÚDE, 2014).

Deste modo, para verificar estas hipóteses, seria necessário coletar dados mais direcionados para o tipo de instruções sobre alimentação que as crianças recebem, tanto na rede pública quanto na rede escolar. 
O hábito de ter uma alimentação saudável não é meramente uma escolha individual, visto que sofre influência econômica, cultural e social, que pode afetar de maneira negativa ou positiva na adoção de padrões alimentares. Neste estudo, verificou-se que mais de $80 \%$ das crianças, tanto da escola pública quanto da escola particular, fazem suas refeições na presença dos pais. Segundo uma atual revisão integrativa da literatura, $o$ ato de se alimentar oportuniza as interações sociais e que o indivíduo, ao realizar suas escolhas alimentares, é influenciado por questões afetivas e familiares, mostrando a importância da família estar sempre unida e fazer uma supervisão mais apurada do que a criança consome (SOUZA AA e CADETE MMM, 2017).

Um dado interessante desta pesquisa é que houve maior proporção de crianças obesas na escola pública $(25,7 \%)$ do que na escola particular (15,5\%), não sendo achados estatisticamente significativos. Sabe-se que a obesidade na infância é um importante indicador de obesidade em adolescentes e, posteriormente, em adultos. Essa relação demonstra a necessidade urgente de medidas preventivas realizadas pelas autoridades para formulação de diretrizes para políticas de saúde, para que esse quadro venha ser reduzido (SANTOS DS, et al., 2019). Esses achados mostram-se diretamente ligados à proporção de crianças da escola pública que fazem suas refeições em frente à televisão, sendo maior estatisticamente que a proporção das crianças da escola particular.

Sousa IR et al (2018) analisou como a mídia (i.e. televisão) influência na alimentação infantil e possíveis impactos que podem ocorrer ao longo da vida. O excesso de tempo assistindo televisão impede que as crianças desenvolvam sua capacidade de fazer a melhor escolha nutricional, pois a mídia facilmente influencia por meio de produtos vinculados a personagens, fazendo com que seu público possam se tornar viciadas em hábitos não saudáveis. Assim, as crianças não recebem informações corretas sobre o real valor dos alimentos saudáveis, não associam que uma alimentação saudável está diretamente ligada à saúde e acabam fazendo a escolha errada. As crianças, além de terem uma interatividade maior com a mídia do que os adultos, são a principais influenciadas por esse veículo que, por estarem em formação, são incapazes de processar as imagens fornecidas pela televisão.

Dentre as estratégias para a melhoria da saúde pública, podem se destacar o incentivo à prática de esportes, juntamente com a melhora de hábitos alimentares (SANTOS DS, et al., 2019). Uma boa alimentação é um dos principais determinantes para melhor desempenho em esportes. Os nutrientes ingeridos são utilizados na forma de energia, reparação tecidual, fortalecimento do sistema imunológico e proteção contra elevações dos radicais livres, resultando na melhora do desempenho geral. Hábitos alimentares são essenciais para quem buscar praticar uma atividade/exercício físico, sendo dispensável o uso de suplementos (SOUZA IR e CARGNIN-CARVALHO A, 2018; SOARES TC, et al., 2019).

Este estudo demonstrou que as crianças da escola particular praticam mais esportes que as crianças da escola pública. Apesar de que não houve diferenças significativas entre o tipo de alimento ingerido e a classificação do IMC, estes achados corroboram com os resultados de que $53,5 \%$ que estão na rede particular são eutróficas.

Queiroz DR, et al (2016) realizou um estudo que avaliou 292 crianças entre 3 e 5 anos de idade, matriculadas tanto em escolas públicas quanto particulares, para avaliar suas competências motoras. Os autores concluíram que é necessário um ajuste nos ambientes físicos e nas oportunidades de prática de esportes em diferentes ambientes escolares, seja em escola pública ou particular, sendo necessários que os profissionais responsáveis por esse ciclo de escolarização e seus gestores criem condições estruturais que adequem os projetos políticos pedagógicos e planos de ensino para o desenvolvimento de habilidades motoras fundamentais. Essas habilidades potencializam condições indispensáveis para o engajamento em esportes e o desenvolvimento motor ativo e saudável ao longo da vida.

Neste estudo, foi possível verificar que as crianças, tanto da rede pública quanto da particular, apresentam peculiaridades em relação à hábitos alimentares e prática de esportes. Essas diferenças podem ser explicadas pelo fato de que foi utilizado uma metodologia subjetiva, na qual a própria criança responde as questões levantadas pelos pesquisadores, sendo, portanto, eficiente na identificação precoce de risco 
nutricional e alterações nutricionais. Por esse motivo, é capaz de detectar sinais de desnutrição que podem passar despercebidos ao considerar apenas peso, altura e IMC na avaliação nutricional, como no caso da classificação segundo as curvas da OMS (BARBOSA-SILVA MC e BARROS AJ, 2002; PIMENTA FS, et al., 2018).

Este estudo foi realizado em uma cidade do interior do estado de São Paulo em uma região que apresenta um Índice de Desenvolvimento Humano de 0,78, considerado alto. Deve-se lembrar de que o Brasil é um país com uma heterogenia à respeito dos hábitos alimentares, segundo cada região, e por isso faz-se necessário o acesso da população à informações confiáveis sobre as características e determinantes de uma alimentação saudável, gerando deste modo a autonomia para escolha de refeições mais benéficas à saúde (MINISTÉRIO DA SAÚDE, 2014; COLEONE JD, et al., 2017). Deste modo, este estudo mostra a importância de futuras pesquisas que tenham abrangência nacional sobre os hábitos alimentares de crianças.

\section{CONCLUSÃO}

Em relação as medidas antropométricas das crianças, não houve diferenças significativas entre as crianças da rede pública em comparação as crianças da rede particular. Este estudo demonstrou também que a proporção de crianças que praticam esportes e a frequência dessa prática são maiores nos escolares da escola particular, sendo que as crianças das escolas públicas apresentaram maior proporção que faz refeições frente à televisão. Estes achados são relevantes pois mostra algumas características de hábitos de saúde, relevantes para o crescimento e desenvolvimento da criança, que apresentam diferenças entre as redes de ensino pública e particular, ressaltando que a rede pública deve priorizar e realizar mudanças para minimizar possíveis alterações que comprometerão a criança pela sua vida.

\section{REFERÊNCIAS}

1. ANTUNES M, LUÍS H. Brincar na educação de infância: conceções e intencionalidade educativa dos/as educadores/as de infância. Revista da UIIPS, 2018; 9(1): 126-38.

2. BARBOSA-SILVA MC, BARROS AJ. Avaliação subjetiva: parte 1 - revisão de sua validade após duas décadas de uso. Arquivos de Gatroenterologia, 2002; 39(3): 181-7.

3. CADAMURO SDP, et al. Consumo alimentar e avaliação nutricional: caracterização de escolares no município de MaringáParaná. CINERGIS, 2016; 17(2): 146-9.

4. COLEONE JD, et al. Perfil nutricional e alimentar de escolares matriculados em uma escola municipal. Ciência e Saúde, 2017; 10(1): 34-8.

5. COSTA AR, BARROS TES, KUNZ E. O brincar como construção racional nas escolas de Educação Física. Revista Motrivivência, 2018; 30(53): 196-208.

6. MINISTÉRIO DA SAÚDE. Secretaria de Atenção à Saúde. Departamento de Atenção Básica. Orientações para coleta e análise de dados antropométricos em serviços de saúde: Norma Técnica do Sistema de Vigilância Alimentar e Nutricional - SISVAN. Brasília: Ministério da Saúde, 2011.

7. MINISTÉRIO DA SAÚDE. Guia Alimentar para a População Brasileira. 2ªed. Brasília: Ministério da Saúde, 2014.

8. MINISTÉRIO DA SAÚDE. Pesquisa de Vigilância de Fatores de Risco e Proteção para Doenças Crônicas por Inquérito Telefônico (VIGITEL). Brasil, 2016.

9. MITRE RMA, GOMES R. A promoção do brincar no contexto da hospitalização infantil como ação de saúde. Ciência e Saúde Coletiva, 2004; 9(1): 147-54.

10. PIMENTA FS, et al. Agreement between subjective global nutrituinal assessment and the nutritional assessment of the world health organization. Jornal de Pediatria, 2018; 94(6): 602-8.

11. QUEIROZ DR, et al. Competência motora de pré-escolares: Uma análise em crianças de escola pública e particular. Motricidade, 2016; 12(3): 56-63.

12. RINALDI AEM, et al. Contribuições das práticas alimentares e inatividade física para o excesso de peso infantil. Revista Paulista de Medicina, 2008; 3(26): 271-7.

13. SANTOS DS, et al. Transição nutricional na adolescência: uma abordagem dos últimos 10 anos. Revista Eletrônica Acervo Saúde, 2019; 20: 2-6.

14. SILVA J, et al. Níveis insuficientes de atividades física de adolescentes associados a fatores sociodemográficos, ambientais e escolares. Ciência e Saúde Coletiva, 2016; 23(12).

15. SOARES TC, et al. Efeitos da suplementação das vitaminas $C$ e E na prática de atividade física: uma revisão sistemática. Revista Eletrônica Acervo Saúde, 2019; 11(7).

16. SOUSA JTA, et al. Influência da mídia no consumo alimentar inadequado de crianças e adolescentes como promotora de doenças crônicas: uma revisão da literatura. International Journal of Nutrology, $2018 ; 11$.

17. SOUZA AA, CADETE MMM.O papel das famílias e da escola na formação de hábitos alimentares saudáveis de crianças escolares. Revista Pedagógica, 2017; 19(40): 136-54.

18. SOUZA IR, CARGNIN-CARVALHO A. Consumo de suplementos nutricionais nas academias da cidade de Braço do Norte-SC. Revista Brasileira de Nutrição Esportiva, 2018; 12(70): 213-221. 